\title{
Efficacy of Needle Prick Combined with Shenmai Pain Point Injection for Tennis Player's Wrist Injury Tenosynovitis
}

\author{
T. YANG, C. JIANG ${ }^{1}$ AND Y. MA ${ }^{2,3^{*}}$ \\ Department of Physical, Northeastern University at Qinhuangdao, ${ }^{1}$ Graduate School of Northeastern University at Qinhuang- \\ dao, ${ }^{2}$ Parallel Robot and Mechatronic System Laboratory of Hebei Province, Yanshan University, ${ }^{3}$ Key Laboratory of Advanced \\ Forging \& Stamping Technology and Science of Ministry of National Education, Yanshan University, Qinhuangdao 066004, \\ China
}

\author{
Yang et al.: Needle Prick Combined with Shenmai Pain Point Injection for Wrist Injury Tenosynovitis
}

\begin{abstract}
Treatment for tennis player's wrist injury tenosynovitis with needle prick plus Shenmai pain point injection was observed and then analyzed the patient's clinical outcomes. Grouping mode of random number table was adopted. Test I group and II group covered 1000 patients, which was also the case for the control group. The three groups of patients received different treatments for wrist injury tenosynovitis and all enrolled patients were tennis players. Test I group's 1000 patients were treated with needle prick plus Shenmai pain point injection therapy, test II group's 1000 patients were treated with needle prick therapy, while control group patients were treated with partial closure therapy. Under different therapeutic intervention models, clinical outcomes of the three groups of wrist injury tenosynovitis patients before and after treatment were compared. Seen from the findings, wrist injury tenosynovitis symptoms of all the patients enrolled in the controlled trial were eased in varying degrees after surgery. Seen from clinical treatment efficiency, test I group's clinical treatment efficiency was significantly higher than that of test II group and control group $(p<0.05)$; test II group's clinical treatment efficiency was significantly higher than that of control group $(p<0.05)$; differences between groups was significant, with statistical research value. In the experimental study, we have come to a conclusion that treatment of wrist injury tenosynovitis with needle prick plus Shenmai pain point injection is with significant effect, which is worthy of clinical promotion and reference.
\end{abstract}

Key words: Needle prick, Shenmai pain point injection, wrist injury tenosynovitis, efficiency

Tennis players are prone to wrist injury tenosynovitis in their career (fig. 1). Wrist injury tenosynovitis occurs on sheath of processus styloideus radii. Abductor pollicis longus and musculus extensor pollicis brevis run through tendon sheath at this position which features shallow and narrow groove ${ }^{[1]}$. For tennis players, when there are many palmar oppositions of thumb, flexion and extension movements then two tendons will continuously rub in relatively narrow tendon sheath. Such mechanical stimulation can cause injury inflammation of tendon sheath, which makes tendon sheath narrower, so it is also called narrow tenosynovitis or wrist injury tenosynovitis. The main clinical manifestations of wrist injury tenosynovitis are swelling, pain, weakness of processus styloideus radii of wrist, thickening and hardening of tendon sheath, which will affect patient's normal work and life ${ }^{[2,3]}$. For tennis players, once they suffer from wrist injury tenosynovitis, it will cause a very serious negative impact on their competitiveness and athletic career. Therefore, clinical staff should attach great importance to clinical diagnosis and treatment of tennis player's wrist injury tenosynovitis, actively explore effective treatment methods to improve clinical outcomes of wrist injury tenosynovitis and benefit more such patients. Our clinical staff treated wrist injury tenosynovitis with needle prick plus Shenmai pain point injection, with significant effect achieved, which effectively restored tennis players motor function. Now, specific treatment is reported and summarized below. This clinical study applied controlled trial for observation. 3000 cases of tennis players were selected as research objects, of which 2500 cases of wrist injury tenosynovitis patients were professional tennis players, while the remaining 500 cases of wrist injury tenosynovitis patients were amateur tennis players and tennis enthusiasts. To ensure grouping fairness and accuracy of test results, the 3000 cases of wrist injury

*Address for correspondence

E-mail: mayunfei@ysu.edu.cn 
tenosynovitis patients were divided into three groups, namely, test I group, test II group and control group. Test I group consisted of 600 cases of male patients, 400 cases of female patients; the patients were aged between $22-31 \mathrm{y}$, with average age at $(28.32 \pm 1.35) \mathrm{y}$; the patients course of disease lasted 12-58 d, with average course of $(23.36 \pm 2.54) \mathrm{d}$. Test II group consisted of 590 cases of male patients, 410 cases of female patients; the patients were aged between 23-31 $\mathrm{y}$, with average age at $(28.31 \pm 1.29) \mathrm{y}$; the patients course of disease lasted 12-57 d, with average course of $(23.41 \pm 2.49) \mathrm{d}$. The control group consisted of 600 cases of male patients, 400 cases of female patients; the patients were aged between 23-31 y, with average age at $(28.18 \pm 1.43) \mathrm{y}$; the patients course of disease lasted $12-57 \mathrm{~d}$, with average course of $(23.31 \pm 2.37) \mathrm{d}$. Comparison of general information of three groups of patients with wrist injury tenosynovitis reveals no significant difference in the three groups of patients general information such as number of cases, gender, age, course of disease. Based on statistical treatment, $p$ value is greater than 0.05 , which can be used as observation and data statistics of controlled trial patients. The 1000 patients of test I group were treated with needle prick plus Shenmai pain point injection. Treatment of needle prick: in pressure pain point of affected part, first perform local skin disinfection with Andoful disinfectant ${ }^{[4]}$. Doctor fixed patient's forearm wrist joint with left hand so that patient's forearm wrist joint was in ulnar deviation state. Meanwhile, doctor pushed patient's cephalic vein outward with left thumb, with a needle sized $0.3 \mathrm{~mm} \times 250 \mathrm{~mm}$ gripped in right thumb, index finger. Quickly perform oblique needle technique at patient's pressure pain point, until reaching patient's subcutaneous fascia, which is the location of tendon sheath (fig. 2). Apply quick prick back and forth in all directions of pressure pain point of the affected area including horizontal, vertical, oblique direction. Lift and thrust needle 10 times in average, last $10 \mathrm{~s}$ or so and then pull out the needle ${ }^{[5]}$. After pulling out the needle, doctor should instruct patient to participate in activities such as dorsiflexion, palmar flexion and rotation of wrist and let patient experience whether pain in the affected area significantly subside when needle is pulled out. Clinical studies have shown that the vast majority of patient's pain can be effectively alleviated after prick treatment with the degree of remission between $50 \%-80 \%$. If patient's pain relief is not obvious, re positioning should be done for further needle prick treatment with the method same as described above. Shenmai pain point injection method:
After completion of needle prick treatment, perform injection in regional pain point site with $4 \mathrm{ml}$ Shenmai injection plus $0.5 \mathrm{ml}$ lidocaine injection of $2 \%$ concentration. Then perform conventional pressing hemostasis after injection. The above treatment should be done once a week, with three times as a course of treatment. During the treatment, patient's adverse reactions should be observed. Once abnormality is found, stop treatment in time, find cause of adverse reactions and provide patient with symptomatic treatment. The 1000 cases of wrist injury tenosynovitis patients in test II group were treated with simple needle prick treatment. Specific treatment method, course of treatment followed test I group, which will not be unnecessarily detailed in this section. The 1000 cases of wrist injury tenosynovitis patients in control group were treated with partial closure therapy. Find pressure pain point and induration at processus styloideus radii then take pressure pain point as needle point. Label with gentian violet ${ }^{[6]}$. After conventional topical disinfection, triamcinolone acetonide acetate $20 \mathrm{mg}$ was extracted with $5 \mathrm{ml}$ syringe, to be injected into the tendon sheath with lidocaine $2 \mathrm{ml}$ of $2 \%$ concentration. For injection, it is required that the drug should be injected into patient's tendon sheath. During the injection, there will be local obvious sense of pain which will also radiate to fingertip and wrist. Patients receiving injections will feel significant resistance to injection and a sense of rupture, which shows that the drug has been injected into the tendon sheath ${ }^{[7,8]}$. The treatment should be provided once a week, with three times as a course of treatment. Among test I group patients, there were 953 effective cases, 47 ineffective cases, with clinically effective rate at $95.30 \%$. Among test II group, there were 908 effective cases, 92 ineffective cases, with clinically effective rate at 90.08 $\%$. Among control group, there were 884 effective cases, 116 ineffective cases, with clinical effective rate at $88.40 \%$. In the course of this clinical study, we referred to related content of "common traditional Chinese medicine syndrome treatment practices" for clinical diagnostic criteria of wrist injury tenosynovitis: slow onset, patient feels obvious pain in radial side of wrist (fig. 3), feels weak when carrying items; there is significant upheaval and clear sense of tenderness in processus styloideus radii, thumb flexion in the palm and wrist ulnar deviation can cause pain in processus styloideus radii ${ }^{[9]}$. Clinical classification basis of wrist injury patients: acute phase: overall incidence feature of wrist injury tenosynovitis is slow onset, but patients with acute attacks are not ruled out. The clinical 
symptoms of acute phase patients incorporate a number of other features in addition to adherence to the above clinical diagnostic criteria. These features are mainly localized swelling, pain, which can spread to the patient's finger, forearm when severe ${ }^{[10]}$; chronic phase: clinical features of chronic wrist injury tenosynovitis patients are serious swelling of processus styloideus radii, obvious tenderness, intensified pain during passive excessive ulnar deviation of wrist, limited thumb flexion. In the diagnostic process, doctor can diagnose patient by way of trial Finkelstein test. Specific method of operation is to perform ulnar deviation of the wrist and thumb flexion to the palm, so that patient's tendon sheath is in tension, then increase pressure on this part to cause pain. Impedance of patient's thumb extension will often bring intense pain, with tenderness between processus styloideus radii and bottom of the first metacarpal bone which can spread to patient's thumb and forearm ${ }^{[11]}$. X-ray is primarily used for imageological examination. Through X-ray plain film radiography, fracture, dislocation and other symptoms of injury were excluded ${ }^{[12]}$. Seen from clinical treatment efficiency, test I group's clinical treatment efficiency was significantly higher than that of test II group and control group $(p<0.05)$; test II group's clinical treatment efficiency was significantly higher than that of control group $(p<0.05)$; differences between groups was significant, with statistical research value. This indicates that treatment of wrist injury tenosynovitis with needle prick plus Shenmai pain point injection enjoys significant effect, thus worthy of clinical promotion and reference.

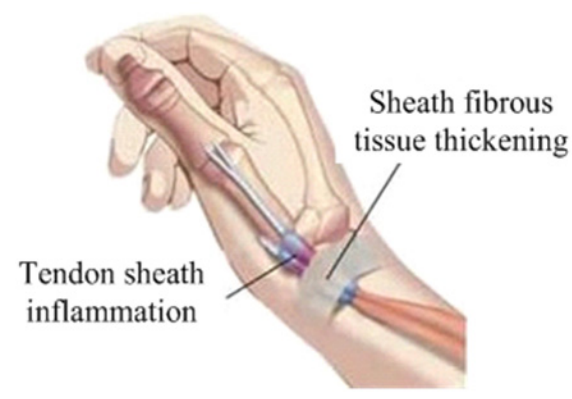

Fig. 1: Schematic diagram of tenosynovitis



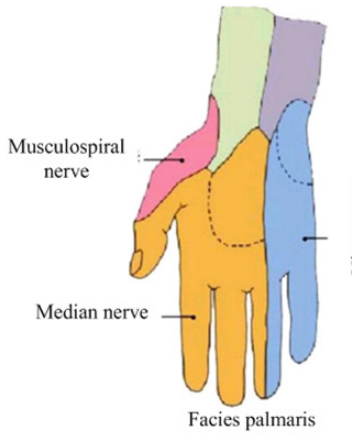

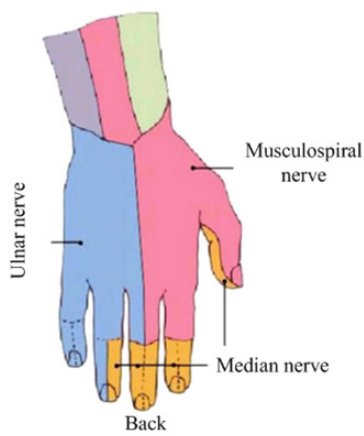

Fig. 3: Schematic diagram of hand nerve

\section{Acknowledgements:}

The research is supported by research and practice project of higher education teaching reform in Hebei Province (No. 2018GJJG426).

\section{Conflicts of Interest:}

The authors declared no conflict of interest.

\section{REFERENCES}

1. Xu XM, Zhang Y, Li H, Sun ZP, Liang C, Geng YZ, et al. Efficacy of needle prick plus Shenmai pain point injection for stenosis tenosynovitis of processus styloideus radii. J New Chin Med 2012;44(3):97-9.

2. Wu P. Clinical comparative study of "easy layer" application and voltaren latex for stenosis tenosynovitis of processus styloideus radii. Nanjing: Nanjing University of Chinese Medicine; 2013. p. 49.

3. Nan DX. Clinical study of acupuncture of Ashi acupoint near muscle region for lateral epicondylitis. Shenyang: Liaoning University of Chinese Medicine; 2013. p. 42.

4. Zhang LH. Clinical observation of distal acupoint selection plus traditional Chinese medicine fumigation for lateral epicondylitis. Heilongjiang University of Chinese Medicine; 2012. p. 52.

5. Chen GH. Clinical efficacy of dragon tiger warring acupuncture combined with massage for early periarthritis of shoulder. Nanjing: Nanjing University of Chinese Medicine; 2014. p. 56.

6. Fu QY, Sun K, Yu B, Chen D, Xie HK, Chen SS, et al. 60 cases of lateral humeral epicondylitis treated by acupuncture at adjacent points of Ashi. Zhejiang J Tradit Chin Med 2019;54(11):838-9.

7. Zhang GM, Zhong XY, Wan CM. Clinical observation on 60 cases of external humeral epicondylitis treated by acupoint injection of bone peptide injection. Psychol Doct 2018;24(14):183-4.

8. Zhang Q, Hou WG, Zhou YL, Li J, Li ZH. Comparison of the clinical efficacy of wrist ankle acupuncture at upper 4 areas and lieque points in the treatment of stenosing tenosynovitis of radial styloid process. Chin J Traditi Med Sci Technol 2017;24(1):100-1.

9. Tian YL. Arthroscopic minimally invasive surgery combined with triamcinolone acetonide in the treatment of stenosing tenosynovitis of flexor tendon. Diet Health 2020;7(25):24-5.

Fig. 2: Schematic diagram of tendon sheath 
10. Sun C, Feng KF, Qi X, Yang YS, Jin HT, Guo HF. The diagnostic value of hand tenosynovitis in rheumatoid arthritis. Chin J Rheumatol 2020;24(7):447-51.

11. Xie HH, Chen MS, Chen RN, Zhao DQ, Li HS, Chen SZ, et al. Analysis on curative effects of manual stretching combined with shock wave in treatment of radial styloid stenosis tenosynovitis and evaluation on wrist function. China Med Pharm 2020;10(3):281-4.
12. Xu HJ, Chen SF, Zhang HM. Ultrasound-guided Local Injection in treatment of hand and wrist tenosynovitis in patients with rheumatoid arthritis. Chin J Med Imag Technol 2019;35(8):1123-7.

This is an open access article distributed under the terms of the Creative Commons Attribution-NonCommercial-ShareAlike 3.0 License, which allows others to remix, tweak, and build upon the work non-commercially, as long as the author is credited and the new creations are licensed under the identical terms

This article was originally published in a special issue, "Clinical

Research in Pharmaceutical and Biomedical Sciences" Indian J

Pharm Sci 2021:83(1)spl issue "207-210". 